\title{
G-SIRS Model with Logistic Growth and Nonlinear Incidence
}

\author{
Ping He $\mathbb{D}^{1,2}$ and Defei Zhang $\mathbb{D}^{2}$ \\ ${ }^{1}$ Department of Mathematics, Anhui Normal University, Wuhu 241000, China \\ ${ }^{2}$ Department of Mathematics, Honghe University, Mengzi 661199, China
}

Correspondence should be addressed to Defei Zhang; zhdefei@163.com

Received 8 June 2020; Accepted 30 June 2020; Published 28 July 2020

Guest Editor: Wenguang Yu

Copyright (c) 2020 Ping He and Defei Zhang. This is an open access article distributed under the Creative Commons Attribution License, which permits unrestricted use, distribution, and reproduction in any medium, provided the original work is properly cited.

We present the stochastic SIRS model in the G-expectation space as follows: $\mathrm{d} X(t)=[r X(1-(X / K))-(\beta X Y /(1+\alpha Y))+$ $\delta Z] \mathrm{d}\langle B\rangle(t)+\bar{\sigma}_{1} X \mathrm{~d} B(t), \quad \mathrm{d} Y(t)=[(\beta X Y /(1+\alpha Y))-(\rho+\vartheta+c) Y] \mathrm{d}\langle B\rangle(t)+\bar{\sigma}_{2} Y \mathrm{~d} B(t), \quad \mathrm{d} Z(t)=[c Y-(\mu+\delta) Z] \mathrm{d}\langle B\rangle(t)+$ $\bar{\sigma}_{3} Z \mathrm{~d} B(t)$, where $\bar{\sigma}_{1}, \bar{\sigma}_{2}, \bar{\sigma}_{3}$ are three intensities of the $G$-Brownian motion and disturb the three variables, and $B(1)$ follows $G$-Normal distribution, namely, $B(1) \sim \mathcal{N}\left(0,\left[\pi^{2}, \bar{\pi}^{2}\right)\right)$. For any initial condition $(X(0), Y(0), Z(0)) \in D^{*}$, we prove the new model admits a unique solution $(X(t), Y(t), Z(t))$ for $t \geq 0$ and the solution $(X(t), Y(t), Z(t))$ satisfies $v(\omega:(X(t)$, $\left.Y(t), Z(t)) \in R_{+}^{3}, t \geq 0\right)=1$.

\section{Introduction}

Liu [1] presented the SIRS model without random perturbation as follows:

$$
\left\{\begin{array}{l}
\mathrm{d} X(t)=\left[r X\left(1-\frac{X}{K}\right)-\frac{\beta X Y}{1+\alpha Y}+\delta Z\right] \mathrm{d} t, \\
\mathrm{~d} Y(t)=\left[\frac{\beta X Y}{1+\alpha Y}-(\rho+\vartheta+\gamma) Y\right] \mathrm{d} t, \\
\mathrm{~d} Z(t)=[\gamma Y-(\mu+\delta) Z] \mathrm{d} t,
\end{array}\right.
$$

where $X(t)$ reflects the susceptible number, $Y(t)$ is the infected number, and $Z(t)$ denotes the recovered number at time $t$. Model (1) took into account logistic growth and nonlinear incidence. The parameters $(\alpha, \beta, \rho, \vartheta, K, \gamma, \delta, \mu)$ in model (1) have practical significance, please refer to reference [1].

The possible region of $(1)$ is $\left\{(X, Y, Z) \in R_{+}^{3}: X+\right.$ $Y+Z \leq K\}=D^{*}$. The basic reproduction number for system (1) is $\mathfrak{R}_{0}=(\beta K /(\rho+\vartheta+\gamma))$.

In the actual environment, various diseases are disturbed by random factors, and there are many models that reflect this stochastic phenomenon, for example, [2-6]. Rajasekar and Pitchaimani [7] assumed this random interference is described by three independent Wiener processes. Specifically, they proposed the following SIRS model:

$$
\left\{\begin{array}{l}
\mathrm{d} X(t)=\left[r X\left(1-\frac{X}{K}\right)-\frac{\beta X Y}{1+\alpha Y}+\delta Z\right] \mathrm{d} t+\sigma_{1} X \mathrm{~d} W_{1}(t) \\
\mathrm{d} Y(t)=\left[\frac{\beta X Y}{1+\alpha Y}-(\rho+\vartheta+\gamma) Y\right] \mathrm{d} t+\sigma_{2} Y \mathrm{~d} W_{2}(t) \\
\mathrm{d} Z(t)=[\gamma Y-(\mu+\delta) Z] \mathrm{d} t+\sigma_{3} Z \mathrm{~d} W_{3}(t)
\end{array}\right.
$$

Peng in $[8,9]$ constructed the interesting G-Brownian motion in nonlinear expectation space, see [10]. Many important properties on $G$-Brownian motion were investigated, for example, [11]. As far as we know, there is no research on model (1) in the nonlinear expectation space. Some notations and concepts used in this paper are similar to those in references $[11,12]$. 


\section{G-SIRS Model}

We consider the stochastic SIRS model in the G-expectation space and propose the G-SIRS model (GSIRSM for short) as follows:

$$
\left\{\begin{array}{l}
\mathrm{d} X(t)=\left[r X\left(1-\frac{X}{K}\right)-\frac{\beta X Y}{1+\alpha Y}+\delta Z\right] \mathrm{d}\langle B\rangle(t)+\bar{\sigma}_{1} X \mathrm{~d} B(t), \\
\mathrm{d} Y(t)=\left[\frac{\beta X Y}{1+\alpha Y}-(\rho+\vartheta+\gamma) Y\right] \mathrm{d}\langle B\rangle(t)+\bar{\sigma}_{2} Y \mathrm{~d} B(t), \\
\mathrm{d} Z(t)=[\gamma Y-(\mu+\delta) Z] \mathrm{d}\langle B\rangle(t)+\bar{\sigma}_{3} Z \mathrm{~d} B(t),
\end{array}\right.
$$

where $\bar{\sigma}_{1}, \bar{\sigma}_{2}, \bar{\sigma}_{3}$ are three intensities of the $G$-Brownian motion, which disturb the three variables, and $B(t)$ satisfies $B(1) \sim \mathcal{N}\left(0,\left[\underline{\pi}^{2}, \bar{\pi}^{2}\right)\right), \widehat{\mathbb{E}}\left[B(1)^{2}\right]=\bar{\pi}^{2}, \widehat{\mathbb{E}}\left[-B(1)^{2}\right]=-\underline{\pi}^{2}$. Note that model (3) has nonlinear incidence. We denote $v(c)=\inf _{P \in \mathcal{Q}} E_{P}\left[1_{c}\right]$ and $V(c)=\sup _{P \in Q} E_{P}\left[1_{c}\right]$, where $\widehat{\mathbb{E}}[\cdot]=\sup _{P \in Q} E_{P}[\cdot]$.

It is very important to prove that the solution $(X, Y, Z)$ of model (3) is of global existence and is nonnegative. We first show system (3) is global and positive. Many asymptotic properties of this system (3) deserve further investigation in the future.

Theorem 1. $\forall(X(0), Y(0), Z(0)) \in D^{*} \quad$ and $\quad t \geq 0$, $(X(t), Y(t), Z(t))$ in (3) are unique and satisfy

$$
v\left(\omega:(X(t), Y(t), Z(t)) \in R_{+}^{3}, t \in[0,+\infty)\right)=1 .
$$

Proof. Since the coefficients of (3) are locally Lipschitz continuous, then $\forall(X(0), Y(0), Z(0)) \in D^{*}$, there exists a local solution $(X(t), Y(t), Z(t))$ on $t \in\left[0, \lambda_{e}\right)$ quasi surely (q.s.), where $\lambda_{e}$ represents the explosion time. To show $\lambda_{e}=$ $+\infty$ q.s., we prove $(X(t), Y(t), Z(t))$ does not explode to infinity in a finite time. Suppose $k_{0}>1$ is large enough such that (s.t) $(X(0), Y(0), Z(0))$ lies in the interval $\left[\left(1 / k_{0}\right), k_{0}\right]^{3}$. For $k \geq k_{0}$, define

$$
\begin{gathered}
\lambda_{k}=\inf \left\{t \in\left[0, \lambda_{e}\right): X(t) \notin\left(\frac{1}{k}, k\right) \text { or } Y(t)\right. \\
\left.\notin\left(\frac{1}{k}, k\right) \text { or } Z(t) \notin\left(\frac{1}{k}, k\right)\right\},
\end{gathered}
$$

where $\lambda_{k}$ is increasing as $k \longrightarrow \infty$. We have $\lambda_{\infty}=\lim _{k \rightarrow \infty} \lambda_{k}$, therefore $\lambda_{\infty} \leq \lambda_{e}$ quasi surely. Suppose we guarantee that $\lambda_{\infty}=\infty$ q.s., then $\lambda_{e}=\infty$ and $v\left(\omega:(X(t), Y(t), Z(t)) \in R_{+}^{3}\right)=1$ q.s. If we assume $0<V\left(\lambda_{\infty}<+\infty\right)$, then there exists a pair of constants $\chi>0$ and $\varepsilon \in(0,1)$ s.t.

$$
V\left(\lambda_{\infty} \leq \chi\right) \geq v\left(\lambda_{\infty} \leq \chi\right) \geq \varepsilon
$$

Then, $\exists k_{1} \geq k_{0}$ s.t.

$$
V\left(\Pi_{k}\right):=V\left(\lambda_{k} \leq \chi\right) \geq \varepsilon, \quad \text { for all } k \geq k_{1} .
$$

Set a function $U_{1}: R_{+}^{3} \longrightarrow R_{+}$by

$$
U_{1}(X, Y, Z)=(X-\ln X)+(Y-\ln Y)+(Z-\ln Z)-3 .
$$

We note the function $g(x):=(x-\ln x)-1 \geq 0$ for any $x>0$. Using the $G$-Ito lemma for the function $U_{1}$, we get

$$
\begin{aligned}
\mathrm{d} U_{1}= & \frac{\partial U_{1}}{\partial x} \mathrm{~d} X+\frac{\partial U_{1}}{\partial y} \mathrm{~d} Y+\frac{\partial U_{1}}{\partial z} \mathrm{~d} Z \\
& +\frac{1}{2}\left[\frac{\partial^{2} U_{1}}{\partial x^{2}}(\mathrm{~d} X)^{2}+\frac{\partial^{2} U_{1}}{\partial y^{2}}(\mathrm{~d} Y)^{2}+\frac{\partial^{2} U_{1}}{\partial z^{2}}(\mathrm{~d} Z)^{2}\right] \\
= & \left(1-\frac{1}{X}\right) \mathrm{d} X+\left(1-\frac{1}{Y}\right) \mathrm{d} Y+\left(1-\frac{1}{Z}\right) \mathrm{d} Z \\
& +\frac{1}{2}\left[\bar{\sigma}_{1}^{2}+\bar{\sigma}_{2}^{2}+\bar{\sigma}_{3}^{2}\right] \mathrm{d}\langle B\rangle(t) \\
= & : \mathscr{L} U_{1} \mathrm{~d}\langle B\rangle(t)+\Theta(X, Y, Z) \mathrm{d} B(t),
\end{aligned}
$$

where

$$
\begin{aligned}
\mathscr{L} U_{1}= & (X-1)\left[\left(\frac{r K-r X}{K}\right)-\frac{\beta Y}{1+\alpha Y}\right]+\frac{\delta(X-1) Z}{X} \\
& +(Y-1)\left[\frac{\beta X}{1+\alpha Y}-(\rho+\vartheta+\gamma)\right] \\
& +\frac{(Z-1) \gamma Y}{Z}-(Z-1)(\mu+\delta)+\frac{1}{2} \sum_{i=1}^{3} \bar{\sigma}_{i}^{2} \\
= & \left(r+\frac{r}{K}\right) X-\frac{r}{K} X^{2}-\frac{\beta X}{1+\alpha Y}+\frac{\beta Y}{1+\alpha Y} \\
& -\rho Y-\vartheta Y-\frac{\gamma}{Z} Y-\frac{\delta}{X} Z-\mu Z \\
& -r+\rho+\vartheta+\gamma+\mu+\delta+\frac{1}{2} \sum_{i=1}^{3} \bar{\sigma}_{i}^{2}, \\
\Theta(X, Y, Z)= & \sigma_{1}^{2}(X-1)+\bar{\sigma}_{2}^{2}(Y-1)+\bar{\sigma}_{3}^{2}(Z-1) .
\end{aligned}
$$

We note that the region $D^{*}=\{X+Y+Z \leq K\}$ and all the parameters are positive, then we have

$$
\begin{aligned}
\mathscr{L} U_{1} & \leq\left(r+\frac{r}{K}\right) X+\frac{\beta Y}{1+\alpha Y}+\rho+\vartheta+\gamma+\mu+\delta+\frac{1}{2} \sum_{i=1}^{3} \bar{\sigma}_{i}^{2} \\
& \leq\left(r+\frac{r}{K}\right) X+\beta Y+\rho+\vartheta+\gamma+\mu+\delta+\frac{1}{2} \sum_{i=1}^{3} \bar{\sigma}_{i}^{2} \\
& \leq \Lambda K+\rho+\vartheta+\gamma+\mu+\delta+\frac{1}{2} \sum_{i=1}^{3} \bar{\sigma}_{i}^{2},
\end{aligned}
$$

where $\Lambda=: \max \{(r+(r / K)), \beta\}$. We denote 


$$
\mathrm{C}=\Lambda K+\rho+\vartheta+\gamma+\mu+\delta+\frac{1}{2} \sum_{i=1}^{3} \bar{\sigma}_{i}^{2} .
$$

Therefore,

$$
\begin{aligned}
\mathrm{d} U_{1}= & : \mathscr{L} U_{1} \mathrm{~d}\langle B\rangle(t)+\Theta(X, Y, Z) \mathrm{d} B(t) \\
& \leq \operatorname{Cd}\langle B\rangle(t)+\Theta(X, Y, Z) \mathrm{d} B(t) .
\end{aligned}
$$

Integrate (13) from 0 to $\lambda_{k} \wedge \chi$,

$$
\begin{aligned}
& U_{1}\left(X\left(\lambda_{k} \wedge \chi\right), Y\left(\lambda_{k} \wedge \chi\right), Z\left(\lambda_{k} \wedge \chi\right)\right) \\
& \quad \leq U_{1}(X(0), Y(0), Z(0))+\mathrm{C} \cdot\langle B\rangle\left(\lambda_{k} \wedge \chi\right) \\
& \quad+\int_{0}^{\lambda_{k} \wedge \chi} \Theta(X(t), Y(t), Z(t)) \mathrm{d} B(t),
\end{aligned}
$$

and take the $G$-expectation,

$$
\begin{array}{r}
\widehat{\mathbb{E}}\left[U_{1}\left(X\left(\lambda_{k} \wedge \chi\right), Y\left(\lambda_{k} \wedge \chi\right), Z\left(\lambda_{k} \wedge \chi\right)\right)\right] \\
\leq U_{1}(X(0), Y(0), Z(0))+\mathrm{C} \cdot \widehat{\mathbb{E}}\left[\langle B\rangle\left(\lambda_{k} \wedge \chi\right)\right] \\
=U_{1}(X(0), Y(0), Z(0))+\mathrm{C} \cdot \bar{\pi}^{2} \cdot\left(\lambda_{k} \wedge \chi\right) .
\end{array}
$$

Note the set $\Pi_{k}(\omega):=\left\{\omega: \lambda_{k}(\omega) \leq \chi\right\}$ and (7), then $V\left(\Pi_{k}(\omega)\right) \geq \varepsilon$ for all $k \geq k_{1}$. We see that the definition of $\lambda_{k}$, then for every $\omega \in \Pi_{k}(\omega)$, there exist at least $X\left(\lambda_{k}(\omega)\right)$ or $Y\left(\lambda_{k}(\omega)\right)$ or $Z\left(\lambda_{k}(\omega)\right)$ equals to $k$ or $(1 / k)$. For example, if $X\left(\lambda_{k}(\omega)\right)=k$ or $X\left(\lambda_{k}\right)=(1 / k)$, then $U_{1}\left(X\left(\lambda_{k}\right), Y\left(\lambda_{k}\right)\right.$, $\left.Z\left(\lambda_{k}\right)\right)=k-1-\ln k+Y\left(\lambda_{k}\right)-1-\ln Y\left(\lambda_{k}\right)+Z\left(\lambda_{k}\right)-1+$ $\ln Z\left(\lambda_{k}\right) \geq k-1-\ln k$, or $U_{1}\left(X\left(\lambda_{k}\right), Y\left(\lambda_{k}\right), Z\left(\lambda_{k}\right)\right)=$ $(1 / k)-1+\ln k+Y\left(\lambda_{k}\right)-1-\ln Y\left(\lambda_{k}\right)+Z\left(\lambda_{k}\right)-1+\ln Z$ $\left(\lambda_{k}\right) \geq(1 / k)-1+\ln k$. Thus,

$U_{1}\left(X\left(\lambda_{k}\right), Y\left(\lambda_{k}\right), Z\left(\lambda_{k}\right)\right) \geq \min \left\{\frac{1}{k}-1+\ln k, k-1-\ln k\right\}$.

From (7) and (14)-(16), we have

$$
\begin{aligned}
& \widehat{\mathbb{E}}\left[I_{\Pi_{k}(\omega)} U_{1}\left(X\left(\lambda_{k}\right), Y\left(\lambda_{k}\right), Z\left(\lambda_{k}\right)\right)\right] \\
& =\widehat{\mathbb{E}}\left[I_{\Pi_{k}(\omega)} U_{1}\left(X\left(\lambda_{k} \wedge \chi\right), Y\left(\lambda_{k} \wedge \chi\right), Z\left(\lambda_{k} \wedge \chi\right)\right)\right] \\
& \quad \leq U_{1}(X(0), Y(0), Z(0))+\mathrm{C} \cdot \widehat{\mathbb{E}}\left[I_{\Pi_{k}(\omega)}\langle B\rangle\left(\lambda_{k} \wedge \chi\right)\right] \\
& \quad \leq U_{1}(X(0), Y(0), Z(0))+\mathrm{C} \cdot \bar{\pi}^{2} \cdot \chi<+\infty,
\end{aligned}
$$

$$
\begin{aligned}
\widehat{\mathbb{E}} & {\left[I_{\Pi_{k}(\omega)} U_{1}\left(X\left(\lambda_{k}\right), Y\left(\lambda_{k}\right), Z\left(\lambda_{k}\right)\right)\right] } \\
& \geq \widehat{\mathbb{E}}\left[\left(\frac{1}{k}+\ln k-1\right) \wedge(k-1-\ln k) \cdot I_{\Pi_{k}(\omega)}\right] \\
= & {\left[\left(\frac{1}{k}+\ln k-1\right) \wedge(k-1-\ln k)\right] \cdot \widehat{\mathbb{E}}\left[I_{\Pi_{k}(\omega)}\right] } \\
= & {\left[\left(\frac{1}{k}+\ln k-1\right) \wedge(k-1-\ln k)\right] \cdot V\left(\Pi_{k}(\omega)\right) } \\
& \geq\left[\left(\frac{1}{k}+\ln k-1\right) \wedge(k-1-\ln k)\right] \cdot \varepsilon .
\end{aligned}
$$

Therefore, from inequalities (17) and (18), we have

$$
\begin{aligned}
& {\left[\left(\frac{1}{k}+\ln k-1\right) \wedge(k-1-\ln k)\right] \cdot \varepsilon} \\
& \quad \leq \widehat{\mathbb{E}}\left[I_{\Pi_{k}(\omega)} U_{1}\left(X\left(\lambda_{k}\right), Y\left(\lambda_{k}\right), Z\left(\lambda_{k}\right)\right)\right]<+\infty .
\end{aligned}
$$

Letting $k \longrightarrow \infty$, we find out inequality (19) is a contradiction. Thus, $V\left(\lambda_{\infty}<+\infty\right)=0$, namely, $v\left(\lambda_{\infty}=+\infty\right)=$ 1 and $v\left(\omega:(X(t), Y(t), Z(t)) \in R_{+}^{3}, t \geq 0\right)=1$.

\section{Discussion}

Although the endemic equilibrium for (1) exists, the endemic equilibrium of the stochastic versions (2) and (3) do not exist. From stochastic stability of Has'minskii [13], Rajasekar and Pitchaimani [7] exemplified that system (2) admits an ergodic stationary distribution. However, in the $G$-expectation space, we first need to obtain the new ergodic stationary distribution theorem similar to the theory of Has'minskii and use it to show the ergodic property for $G$-system (3). We also hope to discuss the disease is extinct for a long time in model (3). We need to find sufficient conditions for extinction of the disease for (3). However, because of the lack of a theorem which is similar to Theorem 1.16 in [14], we cannot get the corresponding results immediately for $G$-system (3). We will investigate the existence of ergodic stationary distribution and the sufficient conditions of extinction for $G$-stochastic system (3) in the future research. By the way, some more realistic and impulsive perturbations models, as well as a nonautonomous case for system (3) are also worth continuing to probe. In addition, numerical simulations for the system will be further investigated.

\section{Data Availability}

No data were used to support this study.

\section{Conflicts of Interest}

The authors declare that they have no conflicts of interest.

\section{Acknowledgments}

This work was funded by Foundations (nos. 11761028, 2015HB061, 2014HB0204, and 2018JS480) and Joint Project of Local Universities.

\section{References}

[1] J. Liu, "Hopf bifurcation analysis for an SIRS epidemic model with logistic growth and delays," Journal of Applied Mathematics and Computing, vol. 50, no. 1-2, pp. 557-576, 2016.

[2] Y. Bin, C. Yongli, W. Kai, and W. Weiming, "Global threshold dynamics of a stochastic epidemic model incorporating media coverage," Advances in Differential Equations, vol. 2018, no. 1, p. $462,2018$.

[3] Q. Liu, D. Jiang, N. Shi, T. Hayat, and A. Alsaedi, "Stationary distribution and extinction of a stochastic SIRS epidemic model with standard incidence," Physica A: Statistical Mechanics and Its Applications, vol. 469, pp. 510-517, 2017. 
[4] X.-B. Zhang, S. Chang, Q. Shi, and H.-F. Huo, "Qualitative study of a stochastic SIS epidemic model with vertical transmission," Physica A: Statistical Mechanics and Its Applications, vol. 505, pp. 805-817, 2018.

[5] Z. Cao, W. Feng, X. Wen, and L. Zu, "Dynamical behavior of a stochastic SEI epidemic model with saturation incidence and logistic growth," Physica A: Statistical Mechanics and Its Applications, vol. 523, pp. 894-907, 2019.

[6] W. Yu, F. Wang, Y. Huang, and H. Liu, "Social optimal mean field control problem for population growth model," Asian Journal of Control, vol. 21, 2019.

[7] S. P. Rajasekar and M. Pitchaimani, "Ergodic stationary distribution and extinction of a stochastic SIRS epidemic model with logistic growth and nonlinear incidence," Applied Mathematics and Computation, vol. 377, Article ID 125143, 2020.

[8] S. Peng, "G-expectation, G-Brownian motion and related stochastic calculus of Itô type," Stochastic Analysis and Applications, Springer, Berlin, Germany, pp. 541-567, 2007.

[9] S. Peng, Nonlinear Expectations and Stochastic Calculus under Uncertainty, Springer, Berlin, Germany, 2019.

[10] S. Peng, "Survey on normal distributions, central limit theorem, Brownian motion and the related stochastic calculus under sublinear expectations," Science in China Series A: Mathematics, vol. 52, no. 7, pp. 1391-1411, 2009.

[11] F. Hu, Z. Chen, and D. Zhang, "How big are the increments of G-Brownian motion?" Science China Mathematics, vol. 57, no. 8, pp. 1687-1700, 2014.

[12] Z. Chen, "Strong laws of large numbers for sub-linear expectations," Science China Mathematics, vol. 59, no. 5, pp. 945-954, 2016.

[13] R. Has'minskii, Stochastic Stability of Differential Equations, Sijthoff and Noordhoff, Alphen aan den Rijn, Netherlands, 1980.

[14] Y. A. Kutoyants, Statistical Inference for Ergodic Diffusion Processes, Springer-Verlag, London, UK, 2004. 